\title{
MODERN TRENDS IN THE SURGERY OF THE COLON
}

\author{
By Sir Hugh Devine, M.S.(Melb.), Hon. F.R.C.S.(Eng.) \\ Melbourne, Australia
}

\section{Carcinoma of the Colon}

\section{Earlier Methods of Operating on the Colon}

If, as a background to the study of the modern trends in the surgery of carcinoma of the colon we take the evolution of the surgery of this organ, we shall better apprecizte its modern drift.

At the turn of the century the feeling was that if an end-to-end anastomosis gave satisfactory results in the small intestine it should be a correct procedure in the large intestine. The method, therefore, came to be the routine for operations on the large intestine which required a resection. The majority of the patients, however, died of peritonitis.

It took some time to dawn on surgeons that the biological conditions of the large intestine were very different from those of the small intestine. The bacterial content was high. The colonic wall in the presence of surgical interference offered a very slender barrier to the migration of germs into the peritoneal cavity. The vitality of the colon wall was very poor since, in most cases, the patients were old and these operations were done for late malignancies. The vascularity of the thin colonic wall was frail and the small vessels were easily occluded by sutures when a piece-meal necrosis occurred. These were the adverse biological conditions the importance of which were not fully recognized.

In the last two decades there has been a gradual evolution in the surgery of the colon in which most of its adverse surgical conditions have been eliminated and its high mortality rate reduced to something within the bounds of surgery of other parts of the alimentary canal. During this evolution, various principles in regard to preoperative, operative and post-operative methods, which are relevant to the practice of modern colonic surgery, have been developed. The use of many of these principles permits of that individualistic surgical treatment of the various phases of colonic disease which is the secret of consistent success in colon surgery. For example, an operator may select that method which will best cope with the particular pattern of colonic disease that he encounters; or he may choose that type of operation which, taking into account his own s'urgical capabilities, is most likely to be successful.

In England, Paul (1895) was one of the first to break away from the accepted end-to-end anastomotic method. He made an anastomosis without sutures; he did this with a crushing clamp in the way that we all know. No peritonitis occurred in I6 out of 17 cases. This extraperitoneal approach, regarded as rather crude at the time, marked a distinct advance. It carried a message, established a principle; it showed one way in which peritonitis could be avoided in the case of a colonic anastomosis. The operation, however, was not radical enough. In the less mobile sections of the colon it did not permit of a radical extirpation of the secondary glands, but the principle on which the operation was based still has a most important use in modern surgery.

In 1928, I93 I, 194I and also in 1948, the author reported the following additions and improvements to this ' spur and enterotome' extraperitoneal method of operation in order to make it comply with the requirements for a completely radical operation for carcinoma of not only the distal, but also of the proximal colon.

I. Mobilization of the whole of the right side of the colon to the mid line for a right-sided lesion (Fig. I), or the whole of the left side of the colon to the same extent for a left-sided lesion (Fig. 2), so that it would be possible to make a complete removal of the mesentery and its glands in each case. This extensive stripping of the colon centrally to its arterial attachments to the aorta is easy and causes little shock.

2. Modification of the spur and enterotome technique which this principle embodies so that it could be applied to join the small intestine to the transverse colon on the right side in the case of an ileocolectomy (Fig. 3), and the small intestine to the mobilized rectum on the left side in a nearcolectomy (Fig. 4). Fig. 5 and Fig. 6 show the method of extraperitoneal operation devised for the removal of carcinoma of the descending colon or of the sigmoid flexure.

3. Extensive modification of the technique so as to $m$ sdify the artificial anus with a view to lessen- 


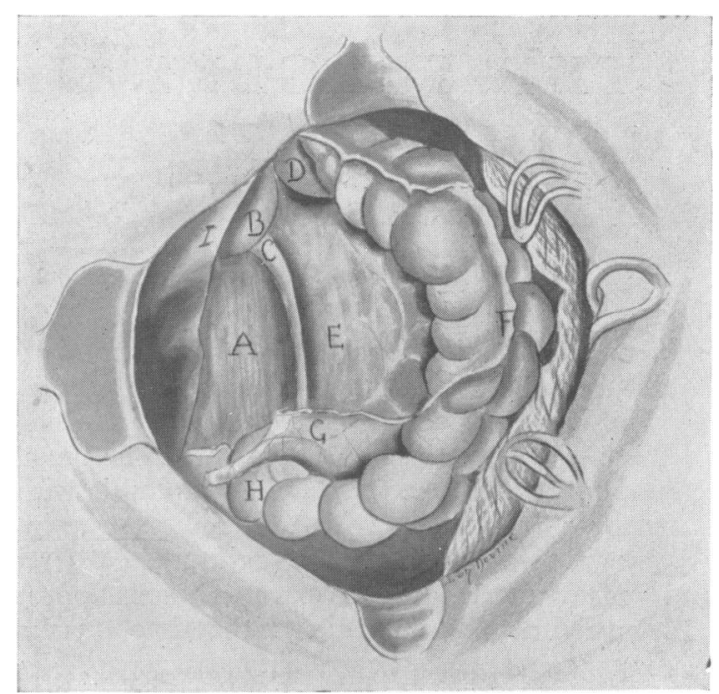

FIG. I.-The ileocolic segment extensively mobilized. $A$, muscles of the posterior abdominal wall; B, kidney; C, ureter; $\mathrm{D}$, duodenum; $\mathrm{E}$, posterior aspect of the mesentery of the proximal colon with vessels; F, mobilized proximal colon; $G$, appendix; H, terminal ileum; I, liver.

ing its unpleasantness and rendering its closure easy and consistent.

\section{Operation on the Defunctioned Left Colon}

There were, however, many cases of disease in the left half of the colon which required resection but in which this extraperitoneal method was impracticable. For these the author originated and practised a two-stage method: 'Operation on a defunctioned distal colon.'

The principles on which this operation is based are that if you completely isolate and deprive the distal colon of its function, which is largely concerned with bacterial activity, it will slowly lose the greater part of this bacterial content; any inflammation in the colonic wall will slowly resolve, faecal lumps can be removed, and this segment of colon can be subjected to direct chemotherapy. A resection can then be carried out and an anastomosis allowed to heal in circumstances where the bowel wall is healthy, at rest, and where there is little infection and no contents. In such circumstances there is practically no danger of peritonitis (Figs. 7 and 8 ).

This method was originally designed for those cases of diverticular obstruction which were associated with extensive colonic cellulitis and

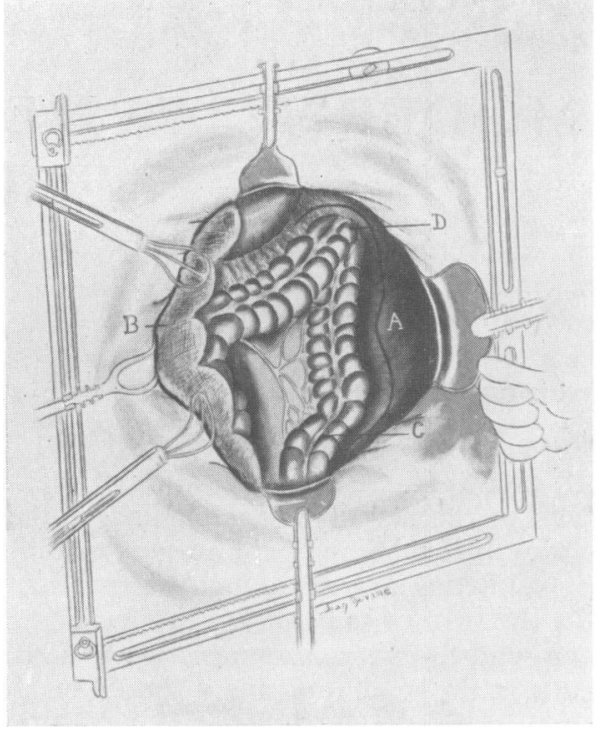

FIG. 2.-Shows insertion of operating frame, clamping on the edges of wound on operation covers. $\mathrm{A}$, incision of peritoneum lateral to descending colon and above the splenic flexure; B, 'parking' with mechanical hands the small intestine into the right side of the abdomen; $C$, sigmoid flexure; $D$, exposure of the splenic flexure.

which, because of the rigid inflammatory colonic walls, did not permit of a safe sutured anastomosis. Its particular application to these cases was that, because of their innocency, the full value of the defunctioning of the bowel could be obtained, for it could be defunctioned for many months. The bowel was defunctioned by using the type of disconnecting anus as shown in Fig. 8-an anus which is small, which completely disconnects the segments of right and left parts of the colon and which is easily closed.

In later years, the author used this method in cases of cancer of the colon with which were associated special surgical difficulties-especially in cases of carcinoma of the descending colon and in the lower part of the sigmoid colon.

The special value of the method is shown by the fact that in particularly complicated cases in which it was used, leaks occurred in the anastomosis, but no peritonitis followed. Indeed, it was difficult to tell that a leak had occurred. Its only evidence was a muco-purulent discharge from the abdominal drainage tube track and the fact that a saline enema came through this track. If the openings in the anastomosis were not big, they closed in time, after which the faecal stream was restored to the defunctioned colon. 


\section{Aseptic Methods of Anastomosis}

In the effort to get better results in the surgery of the colon, there arose another line of thought, namely, the use of various clamps and gadgets with the idea of achieving an aseptic or ' closed' anastomosis in contrast to the usual 'open' anastomosis. With these aseptic methods the names of Rankin, Wangensteen, Gibbons, Hart, Stone, Cope and others have been associated. Of themselves, as methods of operation, these socalled ' aseptic' methods do not seem to have made any epoch-making improvements in the safety of colonic operations. In conjunction with the use of modern chemotherapeutic drugs, however, these methods may have a place in present-day surgery, since, apart from their object of attaining asepsis, they achieve an exactitude in making an anastomosis.

\section{End-to-End or Side-to-Side Anastomoses Com- bined with Caecostomy}

This type of operation is mentioned because it may be regarded as a standard textbook method. As an operation for general application in all the various phases of colonic disease, and unfortified by the use of sulpha and antibiotic drugs, it carried too high a mortality rate. The caecostomy, which is spurless, has little action in defunctioning the colon, for the physiology of the colon is such that most of the faeces will by-pass such an opening. Its only function is to drain the excess of faeces in cases of intestinal obstruction. With the advent of chemotherapy, however, the application of this operation has, today, become wider and its mortality rate much lower.

\section{One-Stage Resection and Anastomosis of the Colon}

The discovery of sulphathaladine and sulphasuccidine, drugs which have a strong selective action on the bacterial content of the colon (Poth, Moore and Miller, I942), marked a turning point in the surgery of the colon. It has led to the revival of the discarded end-to-end anastomosis uncombined with any bowel vent, that is, to a one-stage resection and anastomosis of the colon. Based on the good results of American clinics, this operation threatens to become a surgical vogue. In this attitude of mind, namely a primary resection or nothing, the idealized operation, notwithstanding adverse or unexpected surgical circumstances, lies a definite danger. It must be remembered that its good results-and early published results of advances in surgery by reason of a combination of circumstances are always good-have been obtained by expert surgeons working in highly-organized clinics, specializing in and therefore performing large numbers of this type of operation.

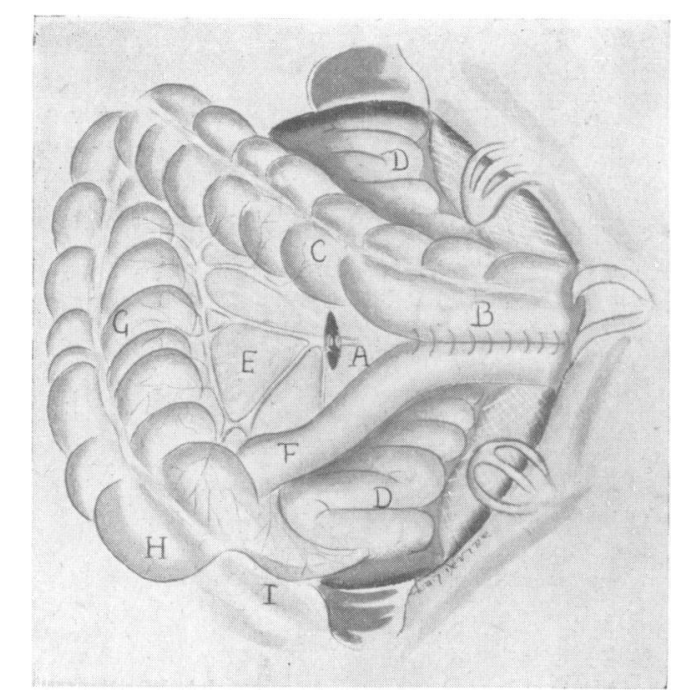

Fig. 3.-Fashioning of the long spur and the division of the ileocolic artery and of the mesenteric leaf. $\mathrm{A}$, division of ileocolic artery; B, long sutured spur; C, proximal part of transverse colon; $D$, small bowel; E, ileocolic mesentery; F, ileum; $\mathrm{G}$, ascending colon; $\mathrm{H}$, caecum; I, appendix.

Many factors have contributed to make this operation possible:-the discovery by Whipple that he could decompress the colon by continuous suction with the Miller-Abbott tube; the advent of chemotherapy, particularly of the sulphonamides, sulphathalidine and sulphasuccidine, drugs which can lower the bacterial content of the bowel to a very great degree in pre-operative and postoperative treatment; the discovery of antibiotics; the organization of nursing teams specially trained in the art of emptying the large bowel; the recognition of the importance of restoring the vitality of the colonic wall by raising the patient's protein, haemoglobin and vitamin levels; the preservation of full vitality of the bowel edges by the avoidance of crushing clamps; improvements in anaesthetic methods; and finally a puinstaking technique in making the anastomosis in which fine mattress stitches are carefully placed and tied to avoid an embarrassment of the circulation. These were the advances and discoveries which made one-stage resection and anastomosis of the colon practicable.

The published mortality rate varies from 5 per cent. to ro per cent. In implementing the concepts of this operation it does not seem to matter whether the anastomosis is made by the 'open' or by the ' closed' method. In point of fact, most surgeons pref $r$ the former.

Prominent among the exponents of this onestage method are: Dickson, McPhee, Wangen- 


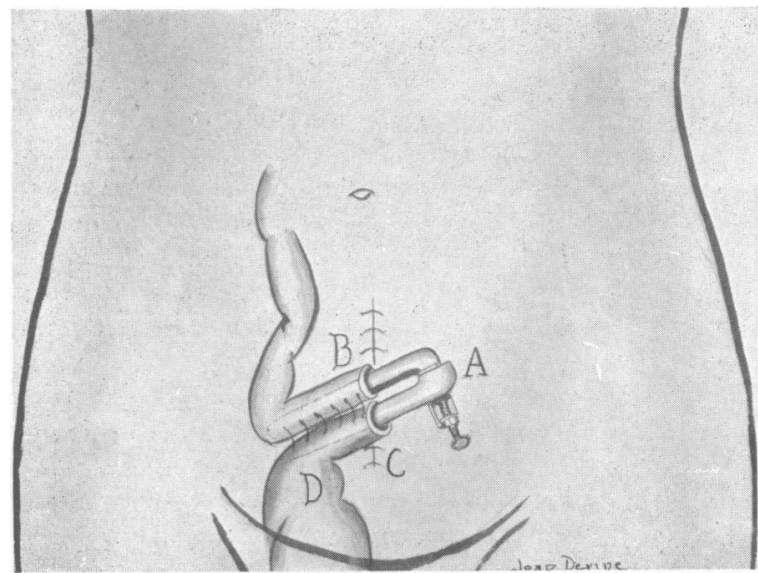

steen, White and Amendover, McNeilly and Lands, Stone and McLauabank, Waugh and Custer.

\section{The Choice of Operation in Colon Surgery}

The approach to the surgery of the colon should be made with the feeling that there can be no routine operation for an organ which presents so many phases of disease, each with its own particular problem; that is, in the choice of operation there are factors in the surgery of the colon other than the septic condition of its contents, which demand individualistic operative treatment and often preclude the ideal primary resection and arastomosis.

The first of these factors is that the healing power of the colonic wall can be inadequate. The vitality of the colonic tissues can be so lowered that the use of sutures in an anastomosis could lead to peritoritis, notwithstanding the use of chemotherapy which can only bring about a very material lowering of the bacterial content, not a complete sterilization. The combination of old age, of the systemic effect on tissues of advanced carcinoma and of the local action on the bowel wall of an acute intestinal obstruction can cause such a devitalization of bowel tissue that it cannot be adequately restored by the most elaborate preoperative preparation. We see this particularly in cases of late and highly cellular carcinoma of the caecum, and in the late growths in the ileocaecal region associated with acute obstruction. Here an extraperitoneal operation (Figs. I and 2) will save the life of a patient who would be lost by the more spectacular prim?ry resection and anastomosis.

David (1943) uses an extraperitoneal operation in all but 5 to 10 per cent. of cases, and Lahey (1946) in all but ro to 15 per cent. of cases. In
FIG. 4.- Shows the ileum joined to the mobilized rectum by the spur and enterotome method. A small fragment of the lower end of the sigmoid is retained in continuity with the rectum to permit the use of this method. A is spur clamp; B the ileal limb of the spur; $C$ the sigmoid limb of the spur; $\mathrm{D}$ the mobilized rectum.

the author's practice the use of ileocolectomy by the extraperitoneal method for over 25 years has given a mortality rate of 5 per cent. Perhaps the most important advantage of this method is that the convalescence is so entirely devoid of incident, so smooth. In our practice there were no cases of local peritonitis from the anastomosis no distensions that gave anxiety, no local abscesse and other complications which are so often seen in the one-stage operation and which frequentl lead to secondary operations.

Lahey (1946) in his last 500 cases had a mortality rate of $2 \frac{1}{2}$ per cent.

On the other hand it must be recognized that ileocaecal growths, in a reasonable early stage in their course even if associated with moderate obstruction, are suitable for primary resection and anastomosis.

A second factor is that the tissues of the colon wall can be acutely and incurably inflamed. In ulcerative colitis, for instance, a sutured anastomosis may not heal or may be followed by a peritonitis as a result of sutures in the highlyinfected tissues. Here no lowering of the bacterial content, even if it could be obtained, would avail. For colectomy in such circumstances we use the extraperitoneal method to anastomose the ileum, through the medium of a terminal segment of the sigmoid, to a fully-mobilized rectum (Fig. 4). In these cases, the worst possible risks, there was not one complication of peritonitis arising from the anastomosis.

The condition of the bowel wall in cas zs of obstructive diverticular tumour associated with extensive cellulitis of the adjoining colon may, too, be a bar to primary resection and anastomosis. So also may the chronic proliferative inflammation of the colonic wall which sometimes follows unskilful pelvic operations with extensive 
injury to the sigmoid. Here primary resection is impossible and good results can only be obtained by 'operation on the defunctioned colon' (Figs. 7 and 8 ). The bowel may have to be defunctioned for 12 months before the inflammation will resolve and the bowel will become supple enough to make a safe sutured anastomosis.

The following case histories are examples of the value of using these conservative methods where they are indicated rather than the more modern primary resection.

Obstructive diverticular tumour and colonic cellulitis.-A woman, aged 50, suffered from an obstructive diverticular tumour in the lower part of the sigmoid colon with much inflammation and rigidity of the adjoining colon. The distal colon was defunctioned for eight months. The operation which was then undertaken showed that the bowel had lost most of its rigidity and that a resection and sutured anastomosis was feasible. However, notwithstanding the most careful technique, on the eighth day it was found that there was a leak in the anastomosis (a saline enema flowed through the abdominal drainage tube track). The only evidence of the leak was a muco-purulent discharge through this track. The abdominal wall was soft and the patient was comfortable. The leak was allowed to heal in the isolated and defunctioned distal colon. In six weeks it had completely healed and then, but not

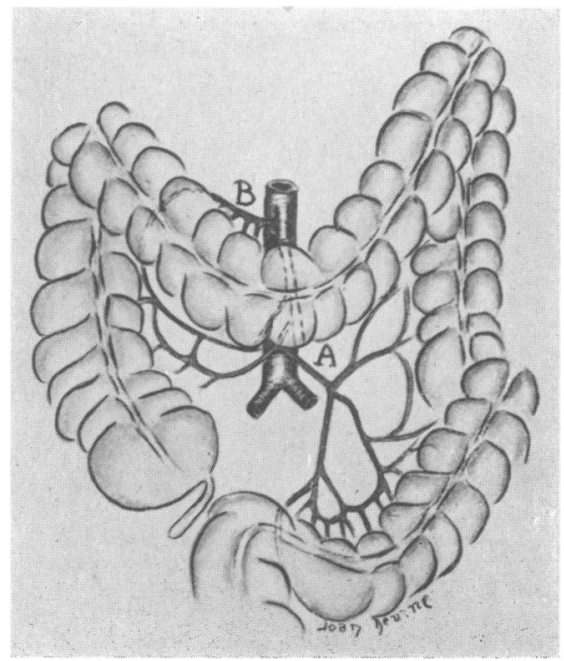

Iic. 5.-Drawn from X-ray studies in the live body. In this it will be seen how the proximal sigmoid will come up to its vascular pedicle $A$, and how the distal part of the transverse colon will swing down to its vascular pedicle B. It will also be seen how much closer together these parts are in life than they are found in anatomical studies. till then, the faecal stream was returned to the distal colon. Had this leak occurred in a primary resection and anastomosis, a peritonitis or localized abscess formation would have followed.

Sigmoid colon injury.- A woman had a gynaecological operation. Following this she developed all the manifestations of peritonitis and was on the verge of death. Finally her abdominal wound opened and from it issued pus and faeces. Eventually, over a period of two years, she became emaciated, exhausted and bedridden, with many abdominal sinuses discharging faeces, with chronically inflamed, rigid and adherent intestines. X-ray showed three large openings in the sigmoid colon. At a preliminary operation to 'soften' the general chronic inflammatory hyperplasia, the distal colon was completely defunctioned. The contents of the colon were cleared out and the distal colon and the sinuses antiseptically treated. The patient was then sent home for nine months. After this period the patient had greatly improved and at the subsequent operation adhesions had largely disappeared, the intestinal wall had become soft and suturable and the faecal fistulae were easily dissected out. The 'defunctioning' had made the operation easy and safe.

A further factor in the choice of operation is the situation of a carcinoma

A growth in the descending colon, at the

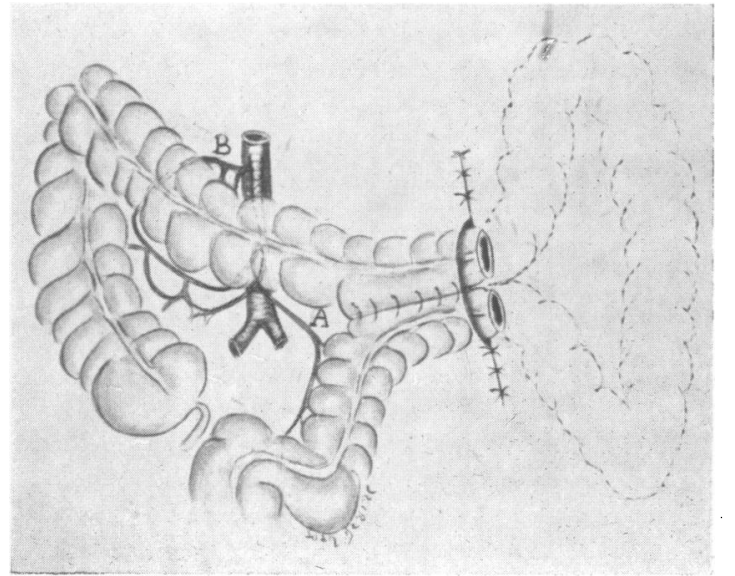

Fic. 6.- Shows how the vascular supply usually permits the formation of a long spur between the distal colon and the proximal sigmoid. Note the long spur at A. The neck of the loop is shown sutured into the parietal peritoneum and the wound. The loop of bowel and mesenteric leaf removed is inincluded in dotted lines. A, ileocolic vascular pedicle; $\mathrm{B}$, mid-colic pedicle. 


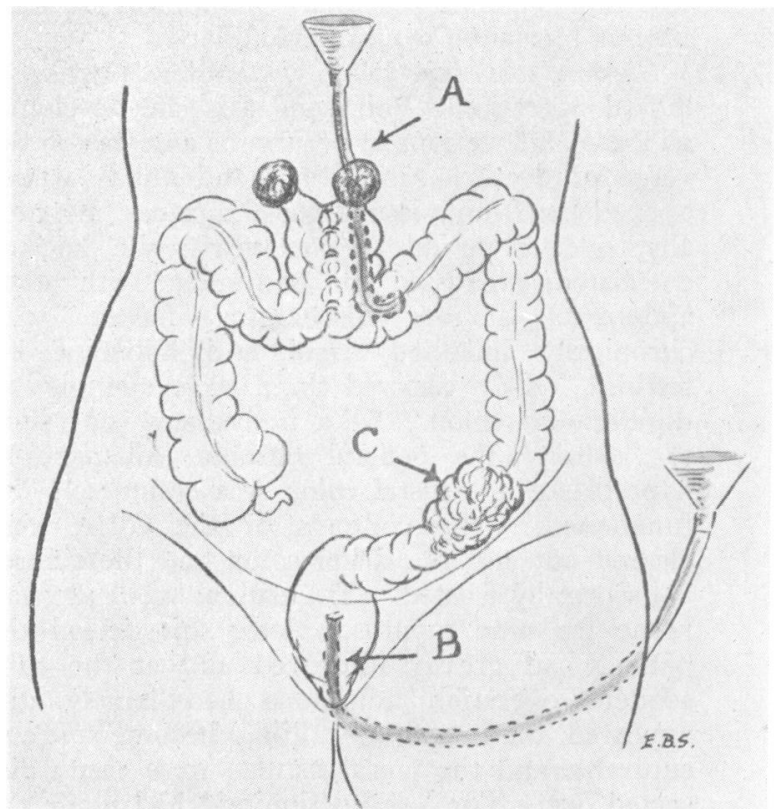

FIG. 7.-Diagram to show the method of complete isolation and defunctioning of the distal colon. $\mathrm{A}$ is the widely separated cut ends of bowel; $\mathrm{B}$, tube in rectum; $\mathrm{C}$, the growth in the distal colon. Funnels show wash-out of isolated bowel from above and below growth.

FIG. 8.- Shows how the small anuses are so separated that no faeces can pass over and reinfect the distal colon after it has been more or less ' debacterialized.'

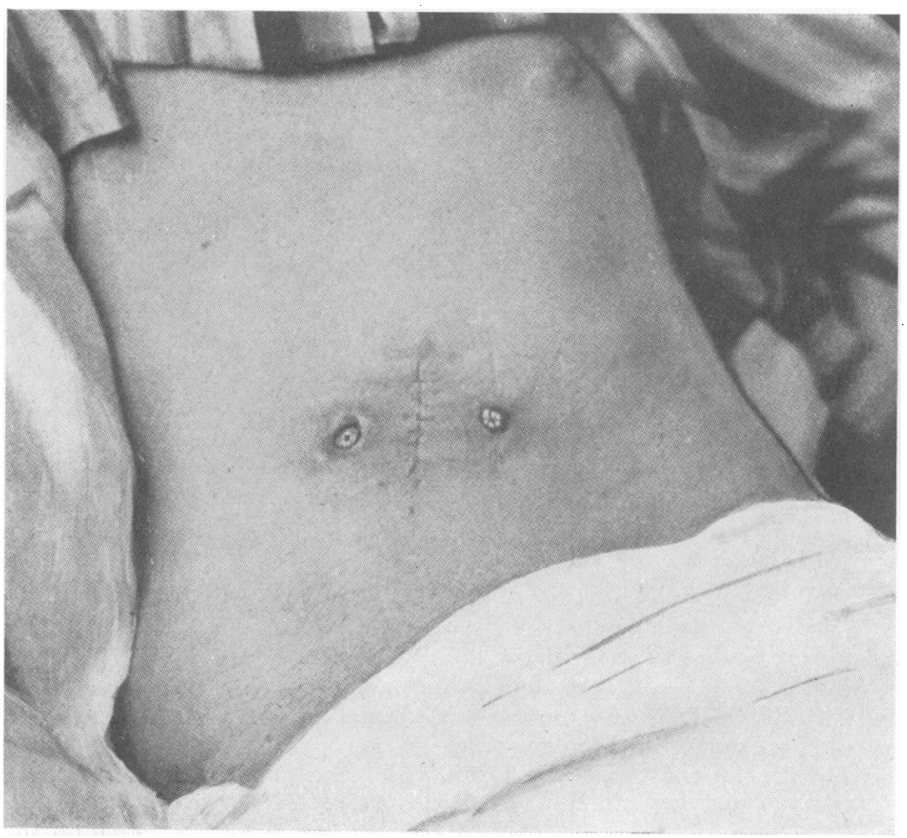


splenic flexure or in the vicinity of the rectosigmoid junction, may make a primary resection and anastomosis inadvisable. Here the extraperitoneal operation pictured in Fig. 4, for splenic flexure and descending colon growths and 'operation on the defunctioned colon' for rectosigmoid growths, are safer procedures than primary resection, and therefore can be said to be a modern trend.

Finally, in the choice of operation, there is the human factor. A medical man, unskilled in surgery, who is confronted with an intestinal obstruction caused by a distal colon carcinomacertainly in our far-out Australian country districts-can not only save his patient's life, but can carry out the first stage of the operation for its removal by placing and making an artificial anus as pictured in Fig. 8. He can carry out the first stage of ' operation on a defunctioned distal colon' at the same time as he relieves the patient's acute intestinal obstruction.

\section{The Choice of Operation for Carcinoma in the Vicinity of the Rectosigmoid Junction}

Here there are two questions. Firstly, should a rectosigmoid resection be performed rather than an abdominoperineal operation ? Secondly, if a rectosigmoid resection is feasible, should a primary resection and anastomosis be carried out, either with or without a bowel vent, or should the more conservative two-stage method, 'operation in a defunctioned and prepared colon,' as shown in Figs. 7 and 8 , be used ? The answer to this problem depends on the particular circumstances of the case. In the earlier years of the author's colon surgery, an abdominoperineal operation was our choice for a carcinomatous lesion in the lower end of the sigmoid. In common with other surgeons we felt that the vascularity of the rectal stump could not be trusted in a rectosigmoid anastomosis. It was felt, however, that the use of an abdominoperineal operation as a routine often inflicted on patients an artificial anus which a rectosigmoid resection could safely have avoided.

Thus, in 1938 , we showed that carefully-selected lesions in the rectosigmoid region could be radically removed by a rectosigmoid resection; that the vascularity of the rectal stump as studied in the live body (Devine, I 94I) could, in most cases, be trusted to make a safe anastomosis; and that the way to ensure safety in this operation, in the case of vascular anomaly, which is not uncommon, was to make an anastomosis in a defunctioned and antiseptically-prepared colon (Figs.7 and 8, and Figs. 9, IO and II). The patients in which this method was used were carefully selected; their growths had to be early and localized. Heyd (I945) and others published cases satisfactorily operated on by this method and used it as a routine.

Since 1940, when Dixon and other American surgeons published a series of good results from primary rectosigmoid resection and anastomosis,
FIG. 9.-Showing illustration of rectosigmoid resection taken from our article in American journal Surgery, 1938. Here the rectum has been mobilized preparatory to resection of the rectosigmoid segment containing the growth.

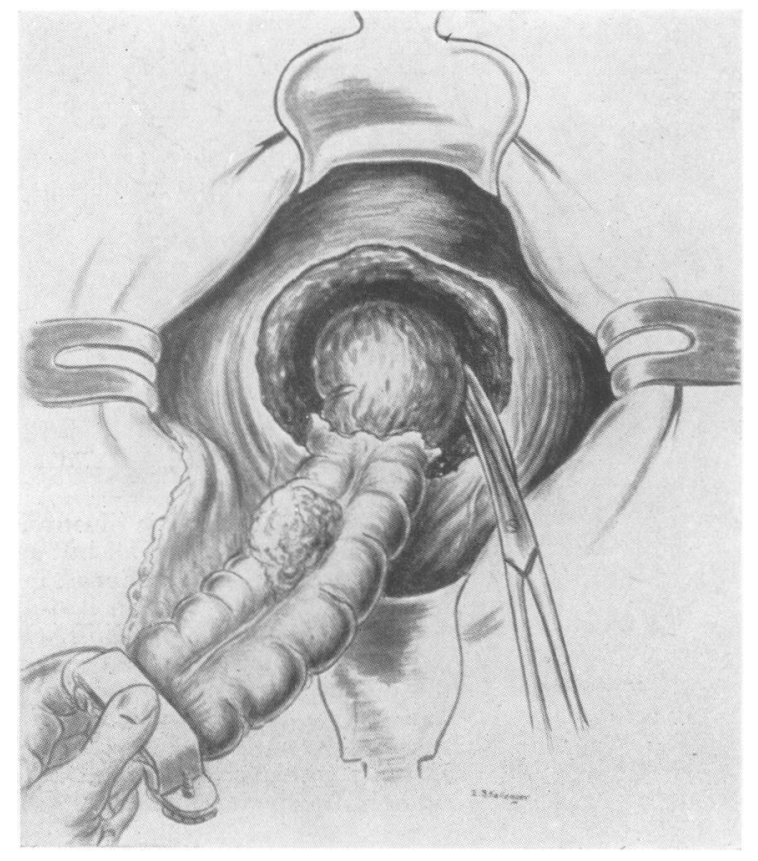



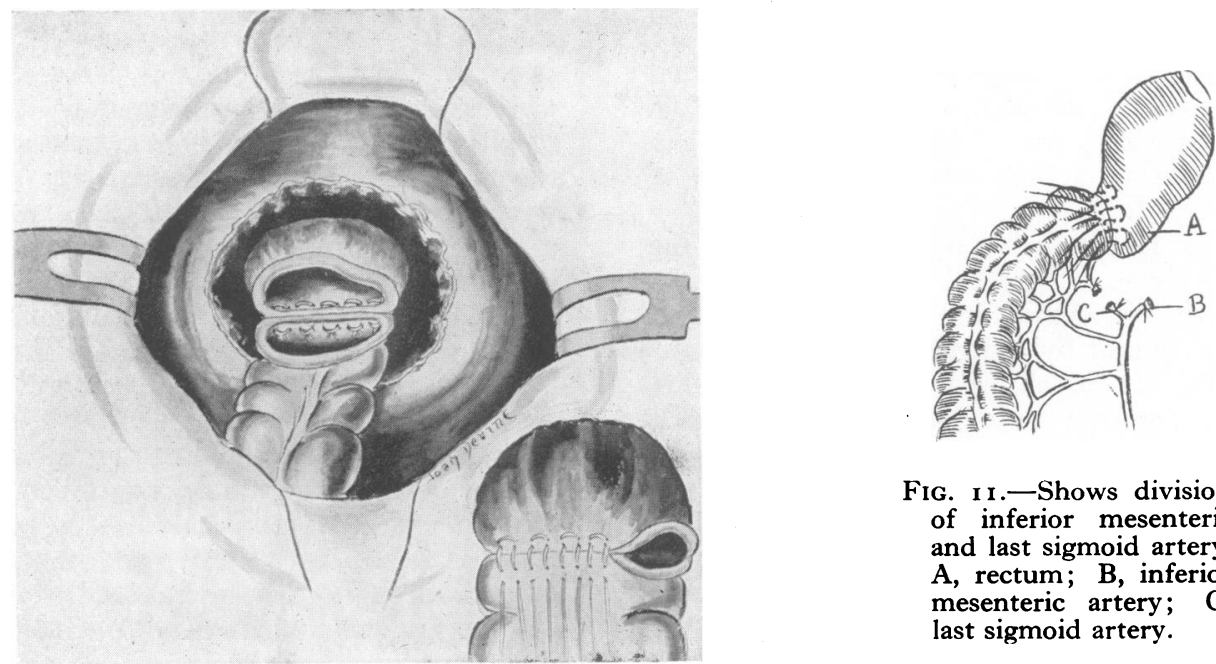

FIG. I 1.-Shows division of inferior mesenteric and last sigmoid artery. $A$, rectum; $B$, inferior mesenteric artery; C, last sigmoid artery.

FIG. 10.-Showing method of making rectosigmoid anastomosis. Inset shows method of inserting mattress sutures.

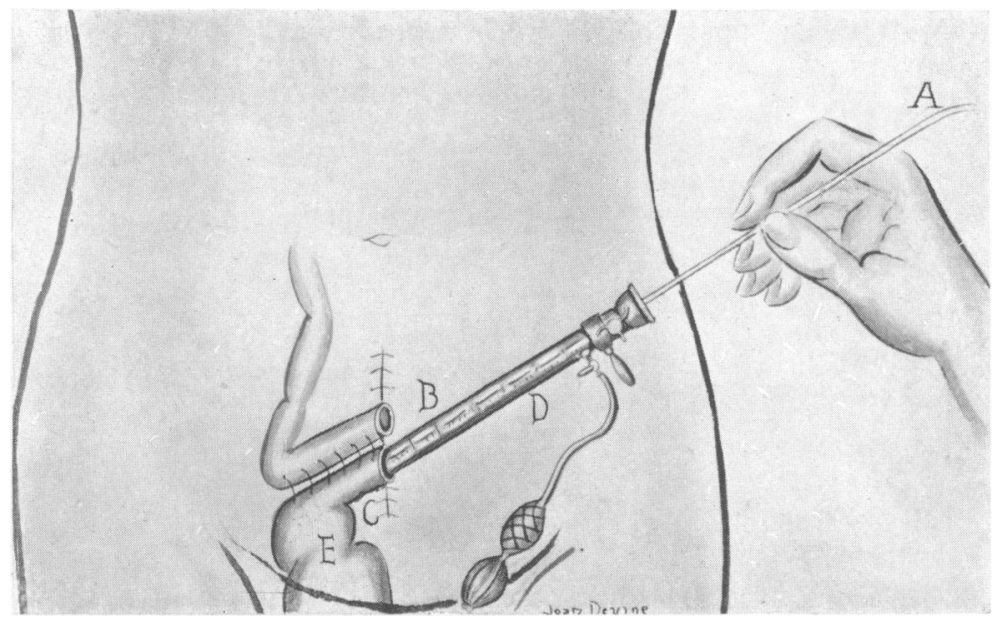

FIG. I2.- This diagrammatic illustration shows a stage in a near-colectomy, in which the colon and all but a fragment of the terminal sigmoid have been removed. The sectioned ileum is joined to the mobilized rectum to make a spur so that an ileo-rectal anastomosis can be made on the spur-and-enterotome principle. Before the ends $\mathrm{B}$ and $\mathrm{C}$ are closed, the polyps in the upper part of the rectum and the sigmoid fragment and also the polyps in the lower part of the rectum (through the anus) are removed by a diathermy snare applied through a sigmoidoscope. $A$ is the snare; $B$ the cut-end of the ileum; $C$ the cut-end of the sigmoid fragment; $\mathrm{D}$ the sigmoidoscope, and $\mathrm{E}$ the mobilized rectum with its attached sigmoid fragment which facilitates the ileo-rectal anastomosis. 
this operation has been practised, in some cases with, in others without, a bowel vent.

Our experience of rectosigmoid resection, covering now about 15 years, would lead us to counsel conservatism in the use of this operation, especially where it is used as a single-stage operation uncombined with a bowel vent. Our reasons are these:-

In the first place, since its use has become rather a vogue, it has been employed for growths which are, perhaps, too far advanced or too active a type of carcinoma. Here its use has been followed by rapid recurrences, sometimes within a year, which shows that this operation was an unwise choice; the method is not as radical as the abdominoperineal operation. In this relation, too, may be quoted Allen of Boston, who has wide experience in colon surgery and is noted for his sound judgment. He writes:- "The trend to save the patient's sphincteric control may lead to inadequate removal and therefore to recurrence.'

In the second place, where a rectosigmoid resection is carried out as a primary resection and anastomosis, consistent results are not obtained. Leaks occur nct so much from septic contents as from vascular deficiencies. From these peritonitis and death may occur, or sinuses and abscess formation may develop and be almost surgically uncurable. Even in the author's own cases in which the sutured rectosigmoid anastomosis was made under the most ideal conditions, leaks occurred. Since however the anastomosis was always made in a distal colon which had been completely isolated, washed out and treated with chemotherapy, nothing untoward happened; no peritonitis followed. There was a discharge of mucus and pus, but the opening, if not too big, always healed, whereafter the faecal current was restored to the distal colon. If, however, the opening did not close because it was too big, and this did occur, it was not a difficult operation to reopen the abdomen and resuture the bowel ends which, by this time, had a well 'determined' circulation. The special point to be made is that if, under these ideal conditions-far more ideal than primary resection and anastomosis protected by the use of sulpha drugs-a carefully-sutured rectosigmoid anastomosis can leak and give trouble, risks from primary rectosigmoid anastomosis will be found to be much greater than reports would have us believe.

In the third place, figures are coming to light which show that retrograde metastases are occurring after rectosigmoid resection for carcinoma below the promontary of the sacrum; and that lesions which are situated at or below the peritoneal reflection have a high incidence of local and hepatic recurrence. In this situation, too, some figures show that where lymph node metastases were found in the surgical specimens, 23 per cent. developed local recurrences within five years.

It is true to write that the more experience the author has had with rectosigmoid resection the more conservative he has become in its use. It will, therefore, be gleaned that his feelings are that primary rectosigmoid resection and anastomosis, with or without a vent, is an operation which demands much experience and sound judgment and is a dangerous operation to put into the hands of the average surgeon. It should be sparingly used.

\section{The Application of One-Stage Resection and Anastomosis}

Having pointed out therefore how many phases of colonic disease do not lend themselves to the practice of primary resection and anastomosis, attention must now be drawn to the considerable field of colonic disease to which such treatmerii is eminently applic :ble.

A. Stephens Graham writes: ' Of 50 surgeons in America with relatively large and prolonged experience in the field of colon surgery, 72 per cent. have performed primary resection and anastomosis in 50 per cent. of cases.' He further writes: 'Of ten surgeons selected for their preeminence in colon surgery, six estimate that they perform primary resection and anastomosis in not more than 25 per cent. of cases.' In these opinions we have some guide to the use we should make of this operation. We should not be too impressed by the many publications which feature small series of successful cases.

Special points in the performance of primary resection and anastomosis are: there should be no complications; any obstruction should be completely decompressed. The intestine must be viable and free from circulatory disturbance. No operation must be attempted until adverse conditions are corrected. There should be a complete mechanical cleansing of the bowel by purgatives and enemas followed by oral sulphasuccidine and sulphathalidine and a low residue diet. There should be careful protection of the wound edges: two rows of inverting mattress sutures; a change of gloves and instruments on the completion of the first row of sutures.

In the case of anastomosis in the vicinity of the rectosigmoid junction, a tube should be passed through the anastomosis. The 'open' operation is preferable because of the accuracy with which. sutures can be placed, and because of the fact that. the full vitality of the wound edges can be retained; in this case rubber-covered clamps should be used to avoid soiling of the operation. field by faecal contents. 
Complications. Some notion of the frequency of complications after this operation may be obtained from published results. One series of 79 cases, taken at random, showed the following complications. There were three cases of peritonitis; six of intestinal obstruction; four of failure of the anastomosis to function; one of urinary fistula; one of general sepsis; one of wound infections; two of phlebothrombosis; and three of coronary thrombosis. In general, a high incidence of complications when compared with those following the extraperitoneal and the twostage operation. Many of these complications entailed secondary operations.

\section{Transplantation of the Proximal Colon into the Anal Canal after Removal of the Distal Colon and Rectum}

Bacon and Smith (1947) report eight cases in which they removed the left half of the colon with the rectum and transplanted the proximal part of the transverse colon into the anal canal with preservation of the sphincter. They write: 'The patients were well satisfied with the continence obtained. There was no mortality in the eight cases. The operation was a formidable one.'

In this type of operation it should be recognized that its potentiality to confer a satisfactory continence is not great because of the destruction of the autonomic nerve supply of the rectum.

\section{Colostomy Closure}

Since the advent of sulphasuccidine and sulphathalidine it has become the custom of some surgeons to close colostomies by an end-to-end anastomosis; that is, by an intraperitoneal instead of an extraperitoneal operation.

\section{Serious Injuries of the Colon}

Late injuries of the colon, especially in the sigmoid region, which sometimes follow - unskilful pelvic operations, provide some of the most difficult operations in colonic surgery. The patients may come under notice with several openings in the abdominal wall pouring faeces and pus, with little or no faeces passing by the rectum, and with all the outward appearance of being very ill. In these cases faecal fistulae with indurated walls lead down through rigid and adherent intestines to the wound in the wall of the injured sigmoid; chronic abscesses form between the intestinal loops in the vicinity of the bowel wound, and intestinal loops adhere to the parietal peritoneum and to one another. The wall of the sigmoid is rigid and thick as the result of longstanding chronic inflammation, and the mucous membrane prolapses through the wound in the bowel and curls over and becomes adherent in such a way that natural healing is impossible.
In these cases the surest way to effect a surgical cure lies in a delayed approach. The distal colon must be completely isolated, 'defunctioned,' by making a disconnecting artificial anus (Figs. 7 and 8). It then receives chemotherapy and is allowed to rest over a period of perhaps 12 months. It will then be found that gradually the chronic inflammation in the walls of the small intestine, in the sinuses and in the sigmoid will resolve, that most of the adhesions will disappear, that the walls of the sigmoid will lose their rigidity and become supple enough to suture, and that the operation becomes reasonably easy.

\section{Diverticulitis}

Babcock (I94I) points out that radical surgery for the early stages of diverticulitis is curative, has a low mortality and should be practised more often than it is.

Diverticulitis with inflammatory infiltration (tumefaction) usually requires surgical intervention. The filling defect when seen in the $\mathrm{X}$-ray is characteristic. It is much longer than that of a malignancy. Diverticula may not show in its neighbourhood because the ostia may be closed by oedema of the bowel wall.

Where a diverticular tumour causes obstruction? part of the obstructive process may be due toㅎ․ oedema and therefore relievable by treatment of the inflammation. Thus, sometimes a defunctioning transverse colostomy may not only relieve the acute obstruction but also it may effect to a large extent a cure of the obstructive process. In our practice we have, in some old and feeble patients who have suffered from an acute obstructive process, been able, after defunctioning the bowel for I2 months, to close their colostomies.

As a rule, however, these diverticular tumours have to be resected. Our method of doing this has already been described in dealing with ' operation on the defunctioned distal colon.'

In cases of diverticulitis where perforation of a diverticulum occurs, a defunctioning transverse colostomy should be made and the segment in which the diverticulum is situated exteriorized. This must be done because the inflammation of the tissue surrounding the perforation makes suture impossible. If the peritonitis remains local the patient recovers, and, if the perforation is not big, it will heal. It may, however, be necessary, where a fistula persists, to resect the affected segment and carry out an end-to-end anastomosis.

Where diverticulitis becomes complicated by a vesico-colic fistula a serious operative problem is presented. The distal colon must be completely isolated and treated with chemotherapy for perhaps 12 months. After this period it will be found 
that it is surprisingly easy to dissect out the fistulous track and close the openings in bowel and bladder.

\section{Polyps}

Helwig's observations show that adenoma is the most common form of polyp and that adenomas occur most frequently in the sigmoid region. David (1943) believes, as does Lahey, that an adenoma is a definitely precancerous lesion; this, too, is our own feeling. It is generally agreed that where a polyp is to be removed locally a biopsy should be made and that the section of tissue should be taken from the base of the polyp. It is also accepted that it is impossible to make a diagnosis of the pathological nature of a polyp except by microscopical examination.

Pedunculated polyps, if few in number, may be removed through one or more colostomies with, of course precautions in regard to malignancy. In order to distinguish between a colonic polyp and a lump of faeces, Dixon, after darkening the room, holds a cold light behind the bowel.

\section{Familial Polyposis}

For this condition, after appropriate preparatory treatment, we remove the colon. If the rectum is extensively involved we remove it at a later stage and leave the patient with an ileostomy. Where the rectum is not extensively affected we endeavour to put off the evil day when the rectum may have to be resected by removing the polyps with a sigmoidoscope, as shown in Fig. 12.

\section{Conclusions}

I. During the last two decades advances in the surgery of the colon have so changed its outlook that it no longer holds the fears for patient and surgeon that it did in earlier years.

2. In these advances early diagnosis of malignancy of the large bowel has been one of the big features.

3. Many of the conservative operative methods which came into use in this period still have a place in modern surgery.

4. The modern methods of primary one-stage resection and anastomosis of the colon, if applied with discretion, mark a spectacular advance in the surgery of the large bowel.

\section{BIBLIOGRAPHY}

BABCOCK, W. W. (I94I), Rev. Gastroenterology, 8, 77. BACON, H. E., and SMİTH, C. H. (1947), F. Int. Coll. Surg., I0, $66 \mathrm{r}$.

DAVID, V. C. (1943), Surgery, 14, 387.

DEVINE, H. B (1928), 'Colon Surgery in the Debilitated,' $\mathcal{~}$. Coll. Surg., Australasia, $1,173$.

DEVINE,' H. B. (I93I), 'Safer Colon Surgery,' The Lancet, I, 627. DEVINE, H. B. (1938), 'Operation on the Defunctioned Colon, Surgery, 3, 165 .

DEVINE, H. B. (I94I), 'Surgery of the Alimentary Tract,' John Wright \& Sons.

DEVINE, H. B., and DEVINE, J. B. (1948), 'Rectum and Colon,' John Wright \& Sons.

DIXON, C. F. (1944), Surgery, 15, 367.

DIXON, C. F., and BENSON, R. E. (1944), Annals of Surgery, 120,562 .
GRAHAM, A. STEPHENS (1949), Surg. Gyn. and Obst., 88, 264 HEYD, C. G. (1945), Am. F. Surg., 67, 479 .

LAHEY, F. H. (1946), S. Clin. N.' America, 26, 6 ro.

MCNEALY, R. W., and LANDS, V. G. (1947), Surgery, 21, 283. MACFEE, W. F. (1947), Annals of Surgery, 126, 125.

PAUL, F.T. (1895), Liverpool Med. Chir. Э., 15, 409.

POTH, E. J. (1945), Surgery, 17, 773.

STONE, H. B., and McLANAHAN, S. (1942), F. Am. Med. Ass. I20, 1362 .

WANGENSTEEN, O. H. (1945), Surg. Gyn. and Obst., 81, I-24. WAUGH, J. M., and CUSTER, M. D. (1945), Ibid., 81, 593

WHIPPLE, A. O. (1940), Surgery, 8, 289.

WHIPPLE, A. O. (1943), Ibid., 14, 321.

WHITE, W. C., and AMENDOLA, F. H. (1944), Ann. Surg., 120 572.

The illustrations of this article are from Sir Hugh and John Devine's book, The Surgery of the Colon and Rectum. They are reproduced here by courtesy of the publishers, Messrs. John Wright and Sons Ltd., Bristol.

\section{RUTHIN CASTLE, NORTH WALES}

A Clinic for the diagnosis and treatment of Internal Diseases (except Mental or Infectious Diseases). The Clinic is provided with a staff of doctors, technicians and nurses.

The surroundings are beautiful. The climate is mild. There is central heating throughout. The annual rainfall is 30.5 inches, that is, less than the average for England.

The Fees are inclusive and vary according to the room occupied.

For particulars apply to THE SECRETARY, Ruthin Castle, North Wales.

Talograme : Caste, Ruthin.

Telephone: Ruthin W 